\title{
Hydraulic fracturing in cells and tissues: fracking meets cell biology
}

\author{
Marino Arroyo $^{1 *}$ and Xavier Trepat ${ }^{2,3,4,5^{*}}$ \\ ${ }^{1}$ Universitat Politècnica de Catalunya-BarcelonaTech, Spain. \\ ${ }^{2}$ Institute for Bioengineering of Catalonia, Barcelona, Spain. \\ ${ }^{3}$ Unitat de Biofísica i Bioenginyeria, Facultat de Medicina, Universitat de Barcelona, \\ Barcelona, Spain. \\ ${ }^{4}$ Institució Catalana de Recerca i Estudis Avançats (ICREA), Barcelona, Spain. \\ ${ }^{5}$ Centro de Investigación Biomédica en Red en Bioingeniería, Biomateriales y \\ Nanomedicina, 28029 Madrid, Spain \\ * Corresponding authors
}

Marino Arroyo, $\mathrm{PhD}$

Associate Professor

Universitat Politècnica de Catalunya

Carrer Jordi Girona 1

08034, Barcelona, Spain

(+34) 934011805

Email: marino.arroyo@upc.edu

Xavier Trepat, $\mathrm{PhD}$

ICREA Research Professor

Institute for Bioengineering of Catalonia

Ed. Hèlix, Baldiri i Reixac, 15-21

08028, Barcelona, Spain

(+34) 934020265

Email: xtrepat@ibecbarcelona.eu 
The animal body is fundamentally made of water. A small fraction of this water is freely flowing in blood and lymph, but most of it is trapped in hydrogels such as the extracellular matrix (ECM), the cytoskeleton, and chromatin. Besides providing a medium for biological molecules to diffuse, water trapped in hydrogels plays a fundamental mechanical role. This role is well captured by the theory of poroelasticity, which explains how any deformation applied to a hydrogel causes pressure gradients and water flows, much like compressing a sponge squeezes water out of it. Here we review recent evidence that poroelastic pressures and flows can fracture essential biological barriers such as the nuclear envelope, the cellular cortex, and epithelial layers. This type of fracture is known in engineering literature as hydraulic fracturing or 'fracking'.
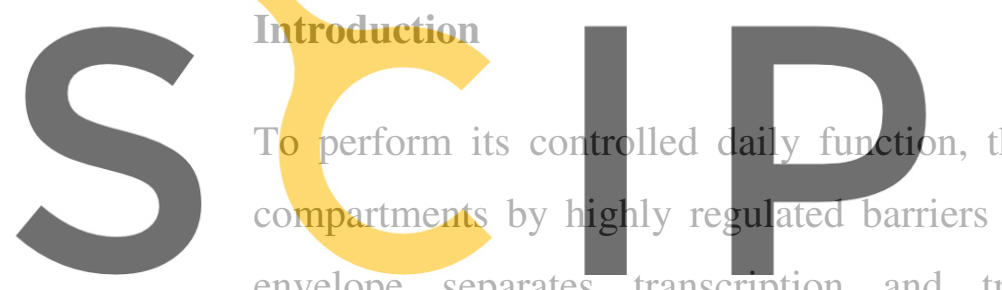

envelope separates transcription and translation,

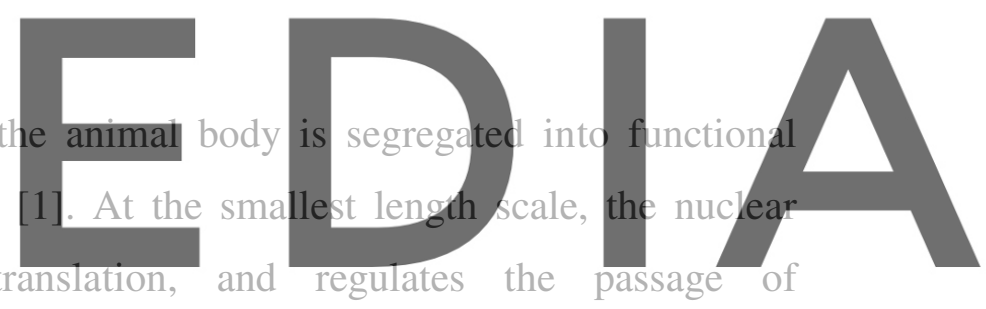

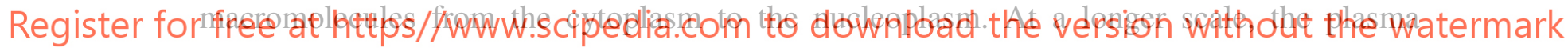

membrane and the underlying cytoskeleton control intracellular composition and provide

cell shape stability. At the supracellular level, epithelial layers control fluid transport, protect organs against the pathogenic attack, and provide structural support to tissues.

Given the crucial role of biological barriers in physiology, it is tempting to think that they have evolved mechanical properties that make them highly resistant to fracture. In contrast with this view, recent evidence has shown that, under some circumstances, biological barriers might undergo fracture routinely: the nuclear envelope breaks as immune cells or cancer cells squeeze through narrow pores $[2 \cdot \bullet, 3 \cdot \bullet]$, the membrane detaches from the cytoskeletal cortex during blebbing [4,5•], and the epithelium exhibits intercellular cracks during physiological levels of stretching [6••]. These three examples share in common that fracture appears to be caused by differences in water pressure and flow. In the engineering literature, this type of fracturing is called hydraulic fracturing or "fracking". Remarkably, 
biological barriers have evolved diverse strategies to heal from hydraulic fracturing within seconds to minutes. Here we review fundamentals of hydraulic fracturing in cells and tissues and we summarize the theory of poroelasticity, which captures flow of water within hydrogels. We then discuss the three examples of hydraulic fracturing mentioned above and the corresponding healing strategies.

\section{Fracture mechanics at a glance}

In the study of the mechanics of inert materials, fracture refers to the separation of a previously cohesive surface within a material as a result of an external action. If this surface is an internal interface in a composite material (here a cell-cell or membrane-network interface), then the phenomenon is called interfacial fracture. A useful framework to

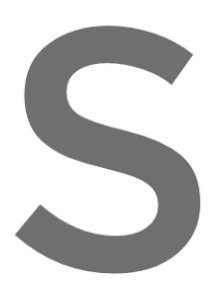
rationalize fracture processes is Griffith's theory [7]. Suppose we apply a force to a
deformable material. The mechanicar work performed on the material, i.e. the applied force
times the resulting displacenent, is stored in it as elastic energy. In an attempt to relax, the
material may develop a crack, thereby releasing stored_elastic energy. However, creating a new free surface in the material costs interfacial energy. According to this theory, fracture

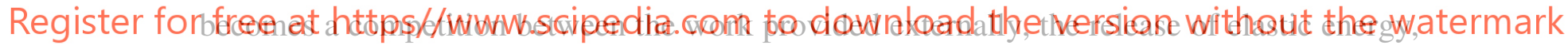

and the energy associated to newly created free surfaces. The external work can involve that of applied forces or that of hydraulic pressure in fluid-filled cracks (Fig. 1). In most real materials, the energy spent in propagating a crack is larger than the intrinsic surface energy, due to various dissipative mechanisms in the so-called process zone near the crack tip, which enhance fracture toughness.

This theory can provide a mechanistic framework for biological processes of interfacial decohesion, but requires accounting for the complexity and active nature of biological materials. Figure 1 suggests two mechanisms for fracture: tensional or hydraulic. In biological tissues, however, both mechanisms are fundamentally coupled because various gels such as chromatin, the cytoskeleton, or the ECM are poroelastic, i.e., they are mixtures of elastic networks swollen by aqueous solvent. Application of forces or deformation changes the chemical potential of water (its pressure) and thus can drive solvent in or out of 
the network, and eventually lead to crack formation or arrest. Conversely, solvent pressure gradients drive solvent flows, which result in mechanical deformation. Because the ability of the solvent to move throughout the dense network is limited, poroelastic processes are time-dependent with a typical relaxation time proportional to the characteristic size of the perturbed region squared (as opposed to the size-invariant relaxation time of viscoelasticity). Interfacial decohesion in biological materials involves complex molecular mechanisms, by which adhesion complexes reorganize under force. For instance, in cellcell doublets under force, tension is transmitted through the actomyosin cytoskeleton to adhesion complexes, eventually leading to failure of the cadherin-cytoskeleton attachment and lateral motion of cadherin-cadherin bonds in the plasma membrane [8]. By contrast, hydraulic fracture of cell-cell interfaces breaks the cadherin-cadherin bonds [6••]. These processes, together with other dissipative phenomena such as inelastic rearrangements of the membrane-cytoskeleton system or poroelastic bulk flows, conform the biological
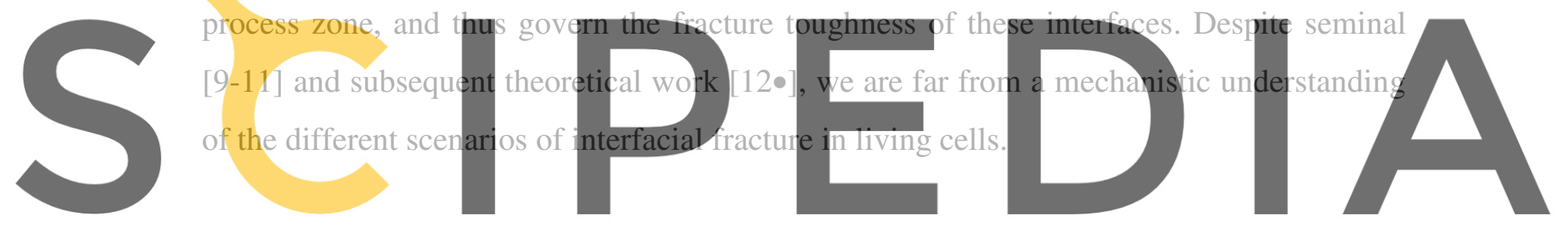

Register for free at https//www.scipedia.com to download the version without the watermark Hydraulic fracturing in cells and tissues

The notion that hydraulic fracturing is intimately linked to cellular function can be illustrated by the phenomenon of cellular blebbing (Fig. 2a,b). Blebs are transient quasispherical protrusions that are ubiquitous in variety of cellular functions such as cytokinesis, motility, spreading and apoptosis [13]. While dynamics can be rather diverse, blebs are relatively short-lived. Typically, a bleb nucleates in an apparently random position of the cell surface, it grows for tens of seconds, and it is reabsorbed within a few minutes. Blebs originate from a myosin-II-mediated delamination of the plasma membrane from the actomyosin cortex [14]. Following delamination, blebs are filled with pressurized fluid from the cytoplasm or from the surrounding extracellular medium [15•]. Blebs have been traditionally associated with convex cellular protrusions, but a recent study showed that high haemodynamic pressure can lead to the so-called inverse blebs, which invaginate into 
the cytoplasm rather than protrude into the extracellular milieu [5•], highlighting the morphogenic role of hydraulic pressure.

While some aspects of blebbing mechanics are well understood, the mechanisms of bleb nucleation remain a matter of debate. An appealing mechanism is hydraulic fracturing caused by a transient contraction of the actomyosin cytoskeleton [4]. This mechanism is based on the idea that the cell interior is poroelastic; a local contraction of the cytoskeleton causes a local pressure buildup that slowly relaxes through water flow in the cytoskeletal network (Fig. 2b). If this network is relatively dilute, water in the contracted region will easily flow away from it without building up a significant pressure differential. However, if the cytoskeletal network is dense, a relatively long-lived pressure transient will develop, the cell membrane will detach from the actin cortex, and a bleb will form. Experimental support to this mechanism includes the observation that blebs can be induced by creating local pressure differences using micropipettes [16], by locally reducing the adhesion energy

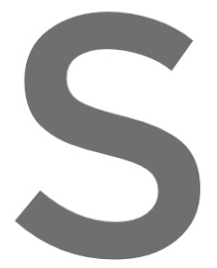
between the cell and its cortex [17], by locally ablating the actin cortex with a laser [18], by
increasing cortical contractility [19], and by depolymerizing the cortex [20]. However,
establishing a definitive causal link between membrane/cortex facture and a local increase in pressure of poroelastic origin remains a remarkable experimental challenge.

Register for free at https//www.scipedia.com to download the version without the watermark More recently, hydraulic fracturing was shown to underlie the formation of intercellular

cracks in epithelial layers $[6 \cdot 0]$. Because the main functions of these layers are to control flow between adjacent body compartments and to protect the body from pathogenic attack, their mechanical integrity is crucial. Maintaining mechanical integrity for a thin layer like the epithelium is particularly challenging in highly dynamic organs, in which cells are routinely subjected to large deformations and pressure differences. In the lung, for example, high stretching levels such as those experienced by patients subjected to mechanical ventilation can exacerbate and even induce acute lung injury [21]. Conversely, high transpulmonary pressure such as that experienced at high altitude can cause pulmonary edema [22].

Epithelial fracture has been traditionally attributed to an excess in tension in cell-cell junctions, cell-matrix junctions, or the plasma membrane [23-25]. As an alternative to this paradigm, Casares et al showed recently that hydraulic fracturing can be at the origin of 
epithelial cracks [6••] (Fig. 2c,d). With a few exceptions, such as early stages of development, epithelial layers are adherent on hydrogel basement membranes of different thickness and composition [26]. According to the theory of poroelasticity [27], compressing the ECM will cause a transient increase in hydraulic pressure and an outflow of water away from the ECM. By contrast, stretching the ECM will cause a pressure drop paralleled by water inflow. If the matrix is covered by an epithelial layer, these differences in transepithelial pressure can lead to fracturing of cell-cell junctions (Fig. 2c). This behavior was recently reported in epithelial clusters of MDCK cells seeded on physiological and synthetic hydrogels matrices [600]. Upon a sudden compression of the clusters, Casares et al observed that virtually every adherens junction was disrupted. This response was not attributable to tensile fracture because tension in the system was lowest at the time of fracture. Instead, the authors showed that intercellular cracks originated from a build-up of hydraulic pressure at the ECM-cell interface. Interestingly, a similar behavior was observed
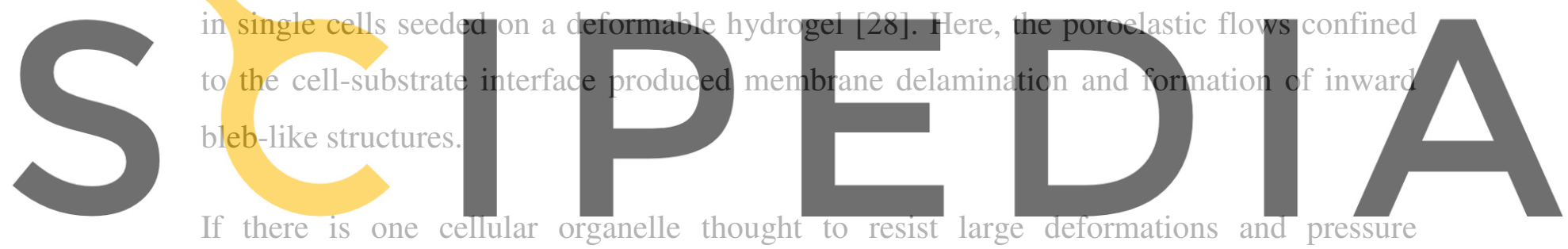

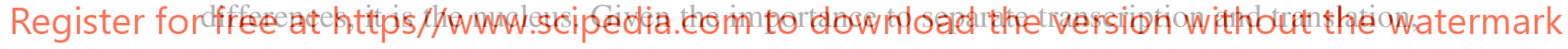

the nucleus has been traditionally assumed to be structured as a tight compartment allowing protein exchange with the cytoplasm only through tightly controlled import and export mechanisms [29]. The only exceptions to this rule were thought to be certain pathological states and the mitotic phase, when the nuclear envelope breaks down to enable binding between condensed DNA and the mitotic spindle [30]. This view was recently called into question by the Piel and Lammerding laboratories who showed that the nuclear envelope breaks when cells squeeze into extremely narrow channels, thereby enabling exchange of fluid and proteins between nucleoplasm and cytoplasm [2••,3••]. Unlike commonly thought, the authors showed that extreme nuclear squeezing might be a relatively usual phenomenon in dendritic cells during immune function and in cancer cells during invasion (Fig. 2e,f). 
When a cell squeezes into a narrow pore, the nucleus divides the cytoplasm into two compartments, a front compartment with relatively low pressure, and a back compartment with relatively high pressure. Application of Laplace's law to this geometry shows that nuclear pressure has an intermediate value closer to the pressure at the back of the cell than that at the front $[2 \bullet \bullet$. This large difference in hydraulic pressure at the front of the nucleus/cytoplasm interface was associated with detachment of the nuclear envelop from the nuclear lamina. Such detachment was proposed to cause the formation of nuclear blebs, which would eventually rupture (Fig. 2e). While differences in hydraulic fracture are thought to be at the origin of nuclear fracture, the role of poroelasticity during this process has not been yet studied. However, it is safe to assume that when a cell migrates into narrow pores, water will be squeezed out from the DNA hydrogel. Besides potential consequences in arrest of nuclear factors, nuclear squeezing will cause a large and sustained pressure of poroelastic origin at the nuclear tip, thereby contributing to nuclear fracture.
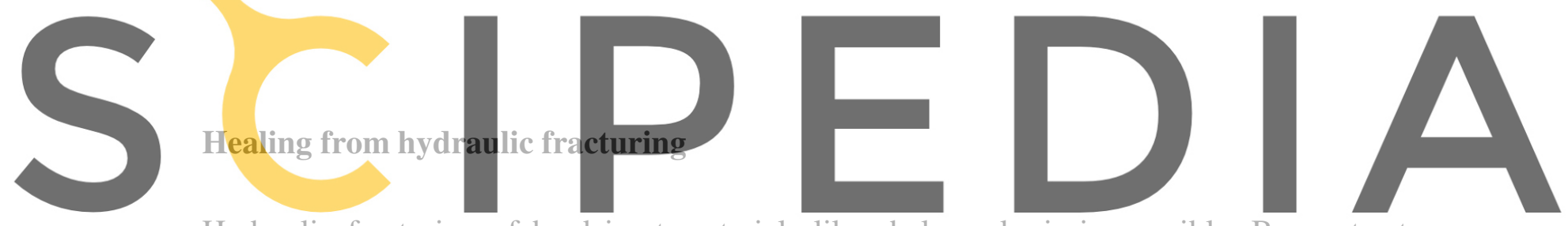

Hydraulic fracturing of hard inert materials like shale rocks is irreversible. By contrast,

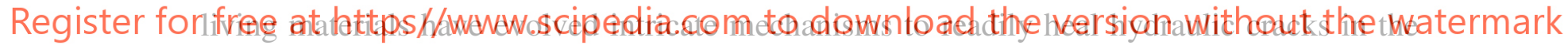

time scale of seconds to minutes. During its growth phase, the surface of a bleb is devoid of actin. With time, however, the cortex rebuilds by sequentially recruiting actin-membrane linker proteins, actin, actin-bundling proteins, regulatory proteins, and finally motor proteins [17]. Assembly of this structure is thought to arrest bleb expansion and, upon contraction, to retract blebs within tens of seconds.

Much like blebs, intercellular cracks resulting from hydraulic fracture seal within a few minutes [6••]. This sealing process is slowed down by myosin inhibition, thus suggesting an analogy with standard purse-string mechanism of wound healing. However, healing of hydraulic cracks displays notable differences with purse-string closure, primarily because water filling intercellular cracks is pressurized. Thus the crack healing mechanism must overcome a hydraulic pressure and force reabsorption of water into the cell cytoplasm or the ECM. To achieve this goal, crack sealing proceeds from the apical to the basal cell 
surface [6••]. Whether cells use specific mechanosensing strategies to sense pressure differences across their plasma membrane to regulate crack sealing is unknown.

Sealing nuclear cracks proceeds through a completely different mechanism than sealing of blebs or intercellular cracks. Raab et al and Denais et al showed that nuclear sealing is mediated by recruitment of the ESCRT-III (endosomal sorting complex required for transport III) machinery to the site of damage $[2 \bullet \bullet, 3 \bullet \bullet]$. This molecular machinery has been implicated in the biogenesis of multivesicular endosomes, virus budding from the plasma membrane of infected cells, cytokinetic abscission, and resealing of the nuclear membrane after mitosis [31]. All these functions share in common the need to constrict, fuse, and/or sever lipid membranes. The mechanism proposed to heal nuclear fracture was analogous to that used to reseal the nuclear membrane after mitosis or to repair the plasma membrane after damage [32-34]. Briefly, a few tens of seconds after fracture of the nuclear envelope, the central ESCRT-III subunit CHMP4B is recruited to the site of damage. CHMP4B
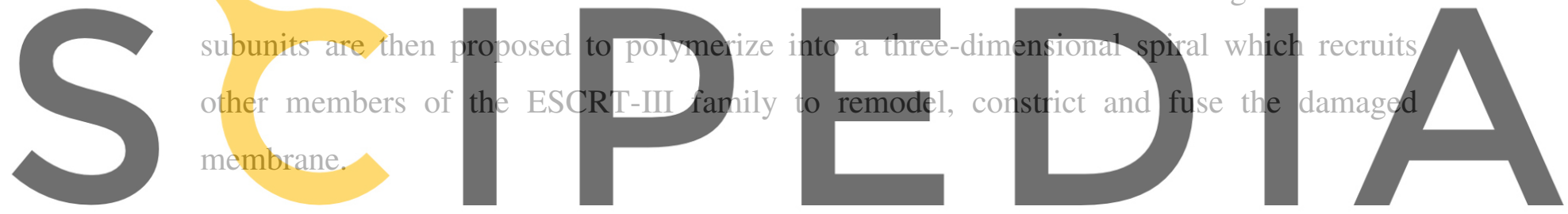

Register for free at https//www.scipedia.com to download the version without the watermark

From fracture to architecture: design guidelines for protective hydrogels

These examples show how concepts from mechanics of materials can provide a framework to understand a number of biological processes involving transient fracture. Conversely, biological architectures can provide new concepts for materials design, such as tough micro-architectured materials made out of brittle constituents inspired in sea shells [35], tough elastomer-hydrogel composites inspired in mammalian skin [36], or polymeric multifunctional self-healing materials [37••]. From a materials perspective, a striking aspect of epithelial sheets subject to stretching is that the poroelastically-driven hydraulic fractures are small and distributed throughout the material, in contrast with most materials that fracture by localizing a single crack. This is a sought after property of structural materials because distributed damage is able to dissipate more energy before failure, and thus lead to enhanced toughness. Lucantonio et al showed that depending of its stiffness, 
porosity or geometry, the presence of a hydrogel adjacent to a material susceptible of cracking fundamentally changes the fracture physics, by making the material tougher and less sensitive to pre-existing flaws [38•]. This insight can help design new artificial materials, understand effects derived from an abnormal or aging matrix, or suggest therapies.

\section{Conclusions and outlook}

Hydraulic fracturing has recently been associated with the rupture of basic biological structures such as membrane/cytoskeleton bonds in blebbing cells, adherens junctions in epithelial monolayers, and the nuclear envelope in cells squeezing through narrow channels. Increasing evidence suggest that these rupture events might be rather common in physiology and disease and should therefore be carefully analyzed. Studying the mechanisms of hydraulic fracturing at the subcellular level poses enormous technical
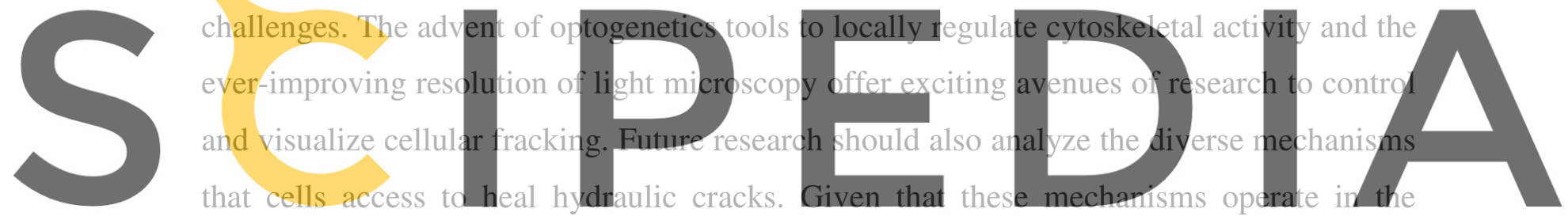
presence of significant pressure gradients and flow they probably differ in fundamental
Register for free at https//WwW.Scipedia.com to download the version without the watermark aspects from currently known mechanisms. Finally, further theoretical effort is needed to

understand how the specific hydrogel composition (chromatin, the cytoskeleton or the ECM) and the molecular makeup of the interface (nuclear membrane-lamins, plasma membrane-cortex, or cell-ECM) determine their mechanical integrity or ability to remodel.

\section{Acknowledgements}

The authors acknowledge L. Casares and E. Latorre for discussions. This work was supported by the Spanish Ministry of Economy and Competitiveness (BFU2015-65074-P to XT, DPI2015-71954-ERC to MA), the Generalitat de Catalunya (2014-SGR-927 to XT, 2014-SGR-1471 to MA), and the European Research Council (CoG-616480 to XT, CoG681434 to MA). 


\section{Figure captions}

Fig. 1: Schematic depiction of a bi-material composite exhibiting interfacial fracture. As external forces or pressures within the crack are applied, the material stores elastic energy, which can be released by propagating a crack at the cost of surface energy and dissipation within the process zone. We distinguish between the ability of the material to resist reversible (elastic) deformation, the stiffness commonly characterized by Young's modulus, and its ability to resist crack growth, the fracture toughness. Fracture toughness is enhanced by dissipative mechanisms occurring at the process zone.

\section{Fig. 2: Examples of hydraulic fracturing in cells and tissues. (a) Membrane blebs have been} proposed to originate from a local contraction (orange) of the cortex (red), which builds up a transient differential of hydraulic pressure in the cortical and subcortical cytoskeleton, CSK (green). This differential is sufficient to disrupt the membrane (mauve) from the
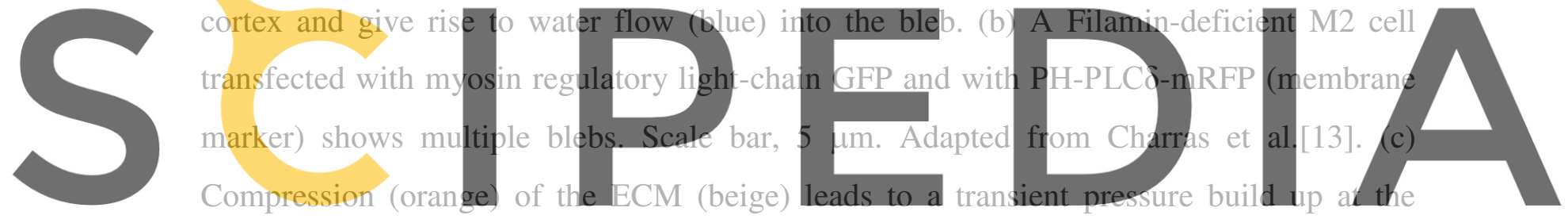
interface with an overlying epithelial monolayer. This pressure differential results in
Register for free at https//WwW.Scipedia.com to download the version without the watermark disruption of adherens junctions, but most tight junctions (green) remain intact. (d)

Compression of a MDCK cluster labelled with lifeact-GFP causes cracks at intercelluiar junctions. These cracks appear as inverse blebs. Scale bar, $40 \mu \mathrm{m}$. Adapted from Casares et al [6]. (e) Nuclear pressure increases as a cell squeezes into extremely narrow channels, possibly leading fracture of the nuclear envelop and exchange of factors between the nucleus and the cytoplasm. (f) A time sequence of a cell migrating in a chemotactic gradient through narrow pores. In the third panel, the nuclear envelope breaks and nuclear localization sequence fused to GFP (NLS-GFP) is released into the cytoplasm. Scale bar, $40 \mu \mathrm{m}$. Adapted from Denais et al [3••]. 


\section{References}

1. Alberts B, Johnson A, Lewis J, Morgan D, Raff M, Roberts K, Walter P: Molecular biology of the cell. Garland Science, (2016).

2. Raab M, Gentili M, de Belly H, Thiam HR, Vargas P, Jimenez AJ, Lautenschlaeger F, Voituriez R, Lennon-Dumenil AM, Manel N, Piel M: Escrt iii repairs nuclear envelope ruptures during cell migration to limit DNA damage and cell death. Science (New York, NY (2016) 352(6283):359-362.

-• This article demonstrates that the nuclear envelope breaks when cells squeeze through narrow channels. Fracture is healed through the ESCRT-III machinery.

3. Denais CM, Gilbert RM, Isermann P, McGregor AL, te Lindert M, Weigelin B, Davidson PM, Friedl P, Wolf K, Lammerding J: Nuclear envelope rupture and repair during cancer cell migration. Science (New York, NY (2016) 352(6283):353-358.

-• This article demonstrates that the nuclear envelope breaks when cells squeeze through narrow channels. Fracture is healed through the ESCRT-III machinery.

4. Charras GT, Yarrow JC, Horton MA, Mahadevan L, Mitchison TJ: Nonequilibration of hydrostatic pressure in blebbing cells. Nature (2005) 435(7040):365-369.

5. Gebala V, Collins R, Geudens I, Phng LK, Gerhardt H: Blood flow drives lumen formation by inverse membrane blebbing during angiogenesis in vivo. Nature cell biology (2016) 18(4):443-450.

- During sprouting angiogenesis, high haemodynamic pressure in the extracellular milieu causes inverse blebs, i.e., quasi-spherical invaginations into the cell body. This article illustrates that hydraulic fracturing is a key enabler of morphogenesis.

6. Casares L, Vincent R, Zalvidea D, Campillo N, Navajas D, Arroyo M, Trepat X: Hydraulic fracture during epithelial stretching. Nat Mater (2015) 14(3):343-351.

- The authors show that hydraulic fracturing underlies the formation of intercellular gaps between epithelial cells. Using the theory of poroelasticity for large deformations they demonstrate how hydraulic fracturing depends on the density and geometry of the extracellular matrix.

7. Zehnder A: Fracture mechanics (lecture notes in applied and computational mechanics). In: Springer, London, (2012).

8. Maître J-L, Berthoumieux H, Krens SFG, Salbreux G, Jülicher F, Paluch E, Heisenberg C-P: Adhesion functions in cell sorting by mechanically coupling the cortices of adhering cells. Science (New York, NY (2012) 338(253-256). 
9. Bell G: Models for the specific adhesion of cells to cells. Science (New York, NY (1978) 200(618-627).

10. Bell GI, Dembo M, Bongrand P: Cell adhesion. Competition between nonspecific repulsion and specific bonding. Biophysical journal (1984) 45(1051-1064).

11. Brochard-Wyart F, de Gennes P-G: Unbinding of adhesive vesicles. Comptes Rendus Physique (2003) 4(281-287).

12. Manakova K, Yan H, Lowengrub J, Allard J: Cell surface mechanochemistry and the determinants of bleb formation, healing, and travel velocity. Biophysical journal (2016) 110(1636-1647).

- In this theoretical paper, a mechano-chemical model of the entire blebbing cycle is developed, including healing, in terms of experimentally measurable parameters.

13. Charras GT: A short history of blebbing. Journal of microscopy (2008) 231(3):466-478.

14. Alert R, Casademunt J: Bleb nucleation through membrane peeling. Physical review letters (2016) 116(6):068101.

15. Taloni A, Kardash E, Salman OU, Truskinovsky L, Zapperi S, La Porta CA: Volume changes during active shape fluctuations in cells. Physical review letters (2015) 114(20):208101.

- The authors provide evidence that, contrary to previous understanding, blebs fill with extracellular fluid rather than cytoplasmic fluid.

16. Maugis B, Brugues J, Nassoy P, Guillen N, Sens P, Amblard F: Dynamic instability of the intracellular pressure drives bleb-based motility. Journal of cell science (2010) 123(Pt 22):3884-3892.

17. Charras GT, Hu CK, Coughlin M, Mitchison TJ: Reassembly of contractile actin cortex in cell blebs. The Journal of cell biology (2006) 175(3):477-490.

18. Goudarzi M, Banisch TU, Mobin MB, Maghelli N, Tarbashevich K, Strate I, van den Berg J, Blaser H, Bandemer S, Paluch E, Bakkers J et al: Identification and regulation of a molecular module for bleb-based cell motility. Developmental cell (2012) 23(1):210-218.

19. Bergert M, Chandradoss SD, Desai RA, Paluch E: Cell mechanics control rapid transitions between blebs and lamellipodia during migration. Proceedings of the National Academy of Sciences of the United States of America (2012) 109(36):14434-14439. 
20. Sedzinski J, Biro M, Oswald A, Tinevez JY, Salbreux G, Paluch E: Polar actomyosin contractility destabilizes the position of the cytokinetic furrow. Nature (2011) 476(7361):462-466.

21. Goligher EC, Ferguson ND, Brochard LJ: Clinical challenges in mechanical ventilation. Lancet (2016) 387(10030):1856-1866.

22. Swenson ER, Bartsch P: High-altitude pulmonary edema. Compr Physiol (2012) 2(4):2753-2773.

23. Vlahakis NE, Hubmayr RD: Response of alveolar cells to mechanical stress. Current opinion in critical care (2003) 9(1):2-8.

24. Trepat X, Grabulosa M, Puig F, Maksym GN, Navajas D, Farre R: Viscoelasticity of human alveolar epithelial cells subjected to stretch. American journal of physiology (2004) 287(5):L1025-1034.

25. Harris AR, Peter L, Bellis J, Baum B, Kabla AJ, Charras GT: Characterizing the mechanics of cultured cell monolayers. Proceedings of the National Academy of Sciences of the United States of America (2012) 109(41):16449-16454.

26. Frantz C, Stewart KM, Weaver VM: The extracellular matrix at a glance. Journal of cell science (2010) 123(Pt 24):4195-4200.

27. Tanaka T, Fillmore DJ: Kinetics of swelling of gels. The Journal of Chemical Physics (1979) 70(3):1214-1218.

28. Kosmalska AJ, Casares L, Elosegui-Artola A, Thottacherry JJ, Moreno-Vicente R, González-Tarragó V, del Pozo MÁ, Mayor S, Arroyo M, Navajas D, Trepat X et al: Physical principles of membrane remodelling during cell mechanoadaptation. Nature Communications (2015) 6(7292).

29. Wilson KL, Dawson SC: Evolution: Functional evolution of nuclear structure. The Journal of cell biology (2011) 195(2):171-181.

30. Hatch E, Hetzer M: Breaching the nuclear envelope in development and disease. The Journal of cell biology (2014) 205(2):133-141.

31. Campsteijn C, Vietri M, Stenmark $H$ : Novel escrt functions in cell biology: Spiraling out of control? Current opinion in cell biology (2016) 41(1-8).

32. Jimenez AJ, Maiuri P, Lafaurie-Janvore J, Divoux S, Piel M, Perez F: Escrt machinery is required for plasma membrane repair. Science (New York, NY (2014) 343(6174):1247136.

33. Olmos Y, Hodgson L, Mantell J, Verkade P, Carlton JG: Escrt-iii controls nuclear envelope reformation. Nature (2015) 522(7555):236-239. 
34. Vietri M, Schink KO, Campsteijn C, Wegner CS, Schultz SW, Christ L, Thoresen SB, Brech A, Raiborg C, Stenmark H: Spastin and escrt-iii coordinate mitotic spindle disassembly and nuclear envelope sealing. Nature (2015) 522(7555):231235.

35. Mirkhalaf $\mathrm{M}$, Dastjerdi aK, Barthelat F: Overcoming the brittleness of glass through bio-inspiration and micro-architecture. Nature communications (2014) 5(3166).

36. Yuk H, Zhang T, Parada GA, Liu X, Zhao X: Skin-inspired hydrogel-elastomer hybrids with robust interfaces and functional microstructures. Nature Communications (2016) 7(12028).

37. Cui J, Daniel D, Grinthal A, Lin K, Aizenberg J: Dynamic polymer systems with self-regulated secretion for the control of surface properties and material healing. Nature materials (2015) 14,1-6.

-• A self-regulating polymer system encapsulating fluid is developed, which is capable to secrete fluid that lubricates its surface or heals the material. It is a remarkable artificial mimic of a complex multifunctional material.

38. Lucantonio A, Noselli G, Trepat X, DeSimone A, Arroyo M: Hydraulic fracture and toughening of a brittle layer bonded to a hydrogel. Physical review letters (2015) 115(18):188105.

- In this theoretical paper, the authors establish the conditions under which a brittle material can be stabilized or fractured by adhesion to a hydrogel. 


\section{applied force}

\section{pressurized fluid-filled crack}

deformed material storing elastic energy

\section{process zone}

\section{material interface}

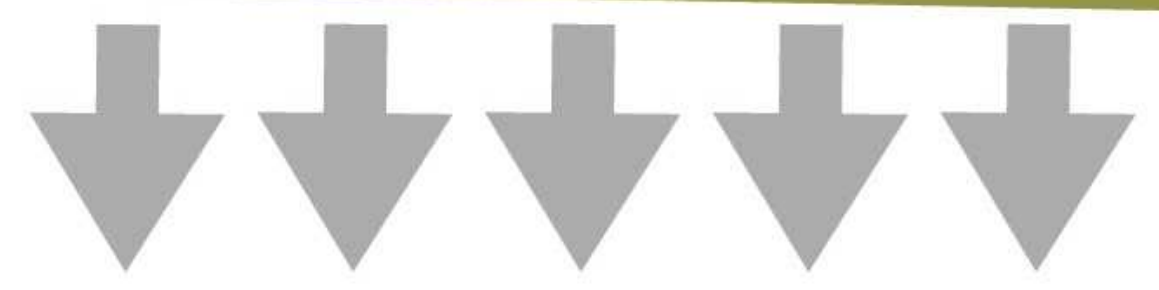


(a) Membrane
Cortex
Subcortical CSK

(b)

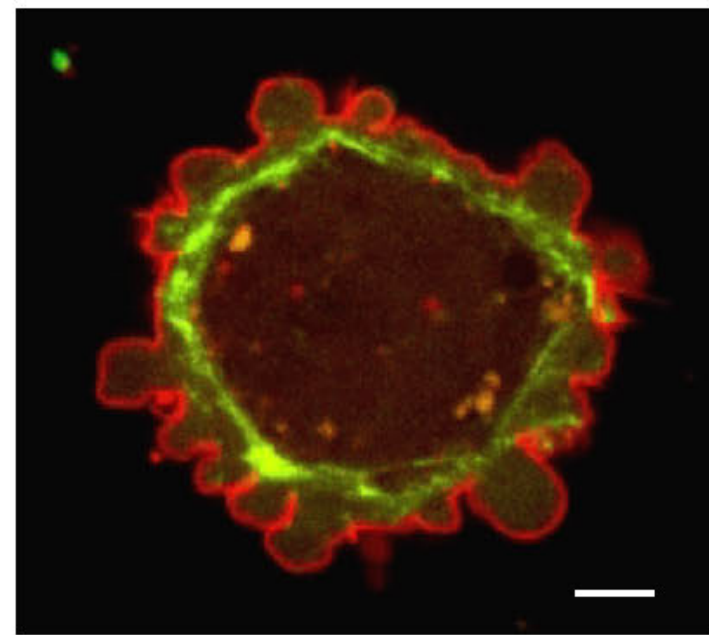

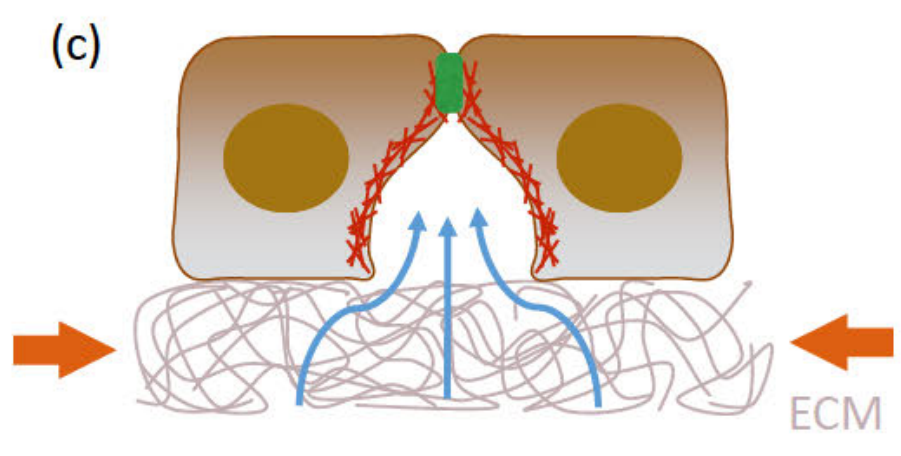

(d)

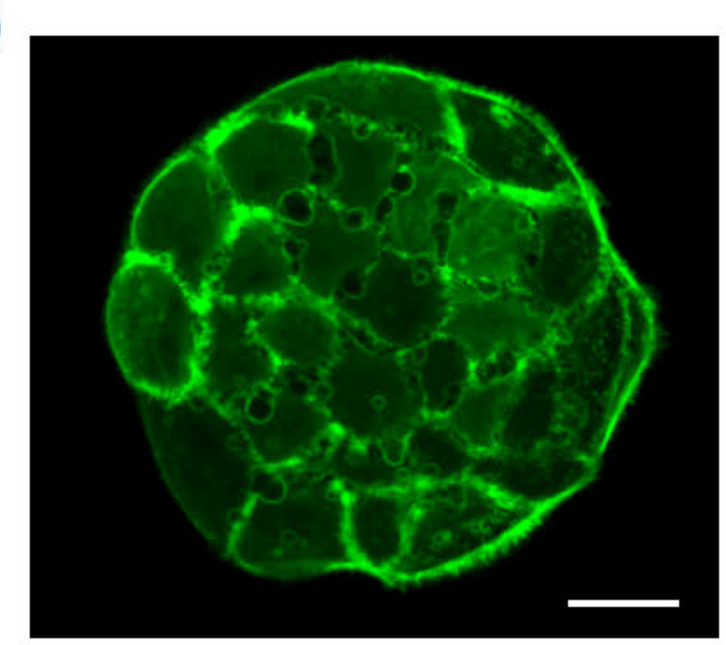

(e)

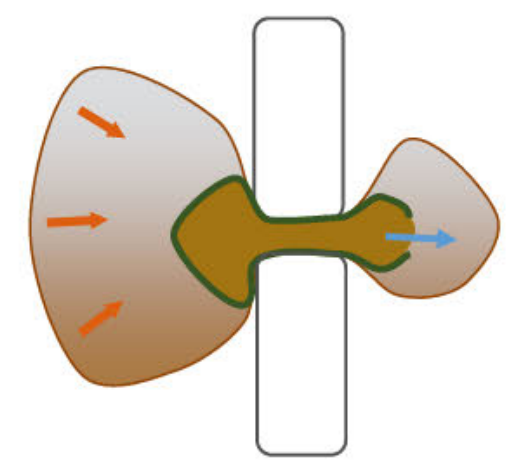

(f)

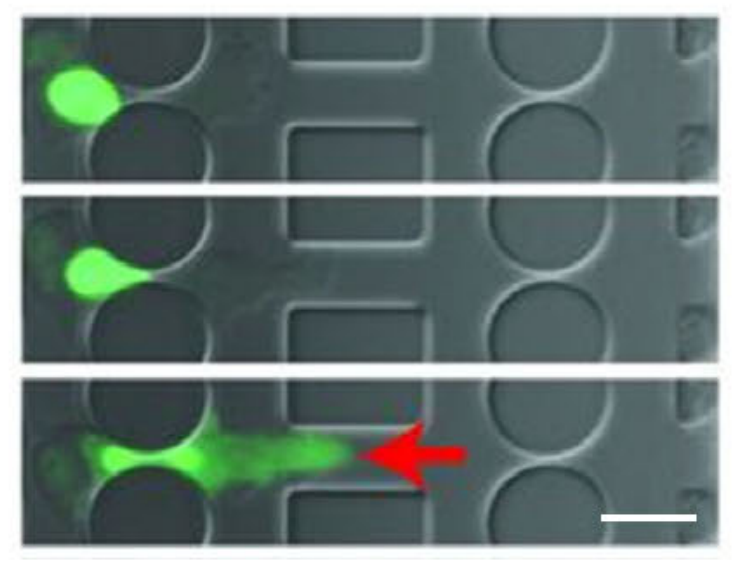

